07

\title{
Соотношения неопределённости числа фотонов и оператора фазы электромагнитного поля для фазовых квантовых суперпозиций когерентных состояний
}

\author{
(C) А.В. Козловский \\ Физический институт РАН им. П.Н.Лебедева, \\ 119991 Москва, Россия \\ e-mail: kozlovskiyav@lebedev.ru
}

Поступила в редакцию 09.07.2019 г.

В окончательной редакции 26.11.2019 г.

Принята к публикации 06.12.2019 г.

\begin{abstract}
Проведено исследование различных соотношений неопределённости для фазы (так называемого оператора Пегга-Барнетта) электромагнитного поля и числа фотонов, тригонометрических функций фазы и числа фотонов, а также тригонометрических функций фазы и фазы поля. Рассмотрено электромагнитное поле в квантовых состояниях фазовых суперпозиций когерентных состояний, представляющих собой общие суперпозиции двух когерентных состояний с одинаковыми модулями и произвольными фазами. Рассматриваются и сравниваются между собой жёсткие соотношения неопределённости (неравенство Коши) и мягкие соотношения неопределённости (неравенства Гейзенберга).
\end{abstract}

Ключевые слова: принцип неопределённости, квантовые флуктуации фазы, эрмитов оператор фазы, суперпозиции когерентных состояний.

DOI: 10.21883/OS.2020.03.49065.235-19

\section{Введение}

Использование неклассических состояний электромагнитного (ЭМ) поля позволяет улучшать фазовую чувствительность интерферометров, широко применяемых для прецизионных измерений. Основанная на соотношении неопределённости $(\mathrm{CH})$ для числа фотонов и фазы поля оценка чувствительности интерферометров (в пределах квантовых флуктуаций) использует предположение о канонической сопряженности операторов этих наблюдаемых. Оценка (скейлинг) уровня флуктуаций проводится с помощью так называемого гейзенберговского предела квантовых флуктуаций $\delta \varphi=\sqrt{\left\langle(\Delta \varphi)^{2}\right\rangle} \sim 1 /\langle n\rangle$ или предела дробового шума $\delta \varphi \sim 1 / \sqrt{\langle n\rangle}[1-3]$, характерного для когерентного состояния ЭМ поля. Использование некоторых неклассических состояний может значительно улучшать фазовую чувствительность интерферометров и позволяет достигать максимального её значения $\delta \varphi \sim 1 /\langle n\rangle$ для большого значения среднего числа фотонов $\langle n\rangle[2,3]$.

Эрмитов оператор фазы поля, предложенный Пеггом и Барнеттом [4-9], позволяет провести точные квантовомеханические расчёты флуктуаций фазы и СН для различных квантовых состояний поля и установить пределы точности интерферометрических измерений, обусловленные квантовыми флуктуациями. Исследования флуктуаций фазы и их возможной минимизации (например, в случае сжатого когерентного состояния) проведены в $[10-13]$.
Квантовое состояние ЭМ поля, представляющее собой суперпозицию когерентных состояний [14-19] относится к числу неклассических состояний поля, не имеющих классического аналога, тогда как квантовое описание поля в когерентном состоянии является наиболее близким к классическому описанию ЭМ поля. Неклассичность суперпозиций когерентных состояний проявляется в виде квадратурного сжатия и субпуассоновской статистики числа фотонов поля, а также плотность вероятности в фазовом пространстве для них оказывается отрицательной или обладает сингулярностями выше чем $\delta$-функции.

В работах [20-22] показана возможность экспериментальных измерений флуктуаций фазы света, используя метод оптической гомодинной томографии совместно с теорией эрмитового оператора фазы Пегга-Барнетта. В $[20,21]$ экспериментально измерены флуктуации фазы света в состоянии сжатого вакуума. В работе [22] измерены правые и левые части СН Гейзенберга для света в когерентном состоянии.

Целью настоящей работы является исследование $\mathrm{CH}$ для фазы и числа фотонов поля, косинуса фазы и числа фотонов поля, а также фазы и косинуса фазы поля. Расчёты проведены в рамках теории Пегга-Барнетта для эрмитового оператора фазы поля. Отдельно для указанного квантового состояния поля рассмотрены $\mathrm{CH}$ Гейзенберга (мягкое соотношение неопределённости, $\mathrm{MCH})$ и жёсткие соотношения неопределённости (ЖСН, неравенство Коши). Найдены условия, при которых ЖСН и МСН значительно различаются между собой. 


\section{ЖСН и МСН для эрмитова оператора фазы}

Рассмотрим операторное неравенство Коши для произвольных эрмитовых операторов поля $\hat{A}$ и $\hat{B}$ следующего вида:

$$
\left\langle(\Delta \hat{A})^{2}\right\rangle\left\langle(\Delta \hat{B})^{2}\right\rangle \geq|\langle\Delta \hat{A} \Delta \hat{B}\rangle|^{2},
$$

где

$$
\Delta \hat{A}=\hat{A}-\langle\hat{A}\rangle, \quad \Delta \hat{B}=\hat{B}-\langle\hat{B}\rangle .
$$

Нетрудно убедиться, что среднее произведения в правой части (1) равно

$$
\langle\Delta \hat{A} \Delta \hat{B}\rangle=\langle\hat{A} \hat{B}\rangle-\langle\hat{A}\rangle\langle\hat{B}\rangle .
$$

Теперь неравенство Коши (1) может быть записано в следующем виде:

$$
\left\langle(\Delta \hat{A})^{2}\right\rangle\left\langle(\Delta \hat{B})^{2}\right\rangle \geq \frac{1}{4}|\langle\Delta \hat{A} \Delta \hat{B}\rangle+\langle\Delta \hat{B} \Delta \hat{A}\rangle+\langle[\hat{A}, \hat{B}]\rangle|^{2} .
$$

Опуская в правой части неравенства (3) два первых слагаемых, мы приходим к МСН для операторов $\hat{A}$ и $\hat{B}$ или к СН Гейзенберга

$$
\left\langle(\Delta \hat{A})^{2}\right\rangle\left\langle(\Delta \hat{B})^{2}\right\rangle \geq \frac{1}{4}|\langle[\hat{A}, \hat{B}]\rangle|^{2} .
$$

Правая часть соотношения (4) определяется только средним квантово-механическим коммутатора операторов.

Отметим, что принцип неопределённости Гейзенберга, выражением которого является неравенство (4), в качестве необходимого условия одновременного точного измерения наблюдаемых системы А и В требует выполнения $\langle[\hat{A}, \hat{B}]\rangle=0$ для операторов наблюдаемых $\hat{A}$ и $\hat{B}$.

Поскольку верно равенство (2), точное (жёсткое) $\mathrm{CH}$ (3) может быть записано также в следующем виде $[23,24]$ :

$$
\left\langle(\Delta \hat{A})^{2}\right\rangle\left\langle(\Delta \hat{B})^{2}\right\rangle \geq|\langle\hat{A}, \hat{B}\rangle|^{2}, \quad\langle\hat{A}, \hat{B}\rangle \equiv\langle\hat{A} \hat{B}\rangle-\langle\hat{A}\rangle\langle\hat{B}\rangle .
$$

Правая часть (5) определяется величиной центрального корреляционного момента операторов $\langle\hat{A}, \hat{B}\rangle=\langle\Delta \hat{A} \Delta \hat{B}\rangle$ и не зависит в явном виде от коммутатора этих операторов.

Из неравенства (5) непосредственно следует, что необходимым условием одновременного точного измерения наблюдаемых системы А и В $\left(\left\langle(\Delta \hat{A})^{2}\right\rangle=0\right.$, $\left.\left\langle(\Delta \hat{B})^{2}\right\rangle=0\right)$ является выполнение равенства $\langle\hat{A}, \hat{B}\rangle=0$, т.е. равенства нулю квантовой ковариации операторов наблюдаемых $\hat{A}$ и $\hat{B}$. Это равенство также является необходимым условием точного измерения одной из наблюдаемых А и В $\left(\left\langle(\Delta \hat{A})^{2}\right\rangle=0,\left\langle(\Delta \hat{B})^{2}\right\rangle \neq 0\right.$ или $\left.\left\langle(\Delta \hat{A})^{2}\right\rangle \neq 0,\left\langle(\Delta \hat{B})^{2}\right\rangle=0\right)$.

Статистическая независимость операторов квадратур поля является достаточным, но не необходимым условием равенства нулю центрального корреляционного момента этих операторов.
Рассмотрим далее в качестве одного из операторов, входящих в СН эрмитов, оператор фазы поля, а также его тригонометрические функции.

В работах [4-9] авторы рассмотрели решение уравнений на собственные функции фазовой переменной в дискретном спектре собственных значений фазы. Расчёты показали, что собственные вектора $\left|\theta_{m}\right\rangle$ операторов фазы поля, рассматриваемые в конечномерном базисе фоковских состояний, для собственных значений фазы

$$
\theta_{m}=\theta_{0}+\frac{2 \pi m}{S+1}, \quad m=0,1, \ldots, S,
$$

где $(S+1)-$ неограниченно большая, но конечная размерность базиса фоковских состояний, $\theta_{0}-$ произвольное число, определяющее интервал изменения собственных значений фазы $\left(\theta_{0} \leq \theta_{m}<\theta_{0}+2 \pi\right)$, составляют полный ортонормированный базис векторов состояний. В работах Пегга и Барнетта $[4,5]$ предложено рассматривать дискретный базис собственных векторов состояний фазы в $(S+1)$-мерном подпространстве фоковских состояний для собственных значений (6) в виде

$$
\left|\theta_{m}\right\rangle=\frac{1}{\sqrt{S+1}} \sum_{n=0}^{S} e^{i n \theta_{m}}|n\rangle .
$$

Эрмитов оператор фазы $\hat{\varphi}_{\theta}$ с собственными значениями $\theta_{m}$ при этом определяется согласно

$$
\hat{\varphi}_{\theta}=\sum_{m=0}^{S} \theta_{m}\left|\theta_{m}\right\rangle\left\langle\theta_{m}|, \quad \hat{\varphi}| \theta_{m}\right\rangle=\theta_{m}\left|\theta_{m}\right\rangle .
$$

Важной особенностью оператора фазы $\hat{\varphi}$, определяемого согласно (8)-(6), является то, что результаты расчётов средних значений и дисперсий фазы поля качественно зависят от выбора параметра $\theta_{0}$. За исключением фоковских $|n\rangle$ и собственных состояний оператора фазы $\left|\theta_{m}\right\rangle$, для которых средние и дисперсии не зависят от $\theta_{0}$, только правильный выбор значения параметра $\theta_{0}$ обеспечивает получение физически осмысленных результатов при расчёте этих средних величин.

Оператор фазы (8) использовался в ряде работ для расчёта средних и дисперсий (флуктуаций) фазы ЭМ поля в различных его квантовых состояниях. Так, в [4-6] найдены средние квантово-механической фазы поля в фоковском $|n\rangle$, когерентном $|\alpha\rangle$ и собственном состояниях фазы $\left|\theta_{m}\right\rangle$ поля. В работах [10-13] проведены расчёты средних значений фазы для двухфотонного (сжатого) когерентного состояния. В $[18,19]$ рассмотрены флуктуации оператора фазы в состояниях суперпозиций $N_{ \pm}(|\alpha\rangle \pm|-\alpha\rangle)$. В работе [25] найдены флуктуации фазы для общих фазовых суперпозиций когерентных состояний.

Векторы квантовых состояний суперпозиции двух когерентных состояний поля в частном случае, когда когерентные состояния $|\alpha\rangle$ и $|\beta\rangle$, составляющие суперпозицию, имеют одинаковые значения амплитуд $n_{\alpha}=n_{\beta}$, 
$n_{\alpha} \equiv|\alpha|^{2}, n_{\beta} \equiv|\beta|^{2}$, но разные значения фазовых углов $\varphi_{\alpha} \neq \varphi_{\beta}$, носят название фазовых суперпозиций. В настоящей работе нами рассмотрены и проанализированы свойства СН для эрмитова оператора фазы ЭМ поля в состояниях фазовых суперпозиций когерентных состояний вида

$$
\begin{gathered}
\left|\Phi_{\alpha, \beta}(\xi)\right\rangle=N_{\alpha, \beta \xi}\left(|\alpha\rangle+e^{i \xi}|\beta\rangle\right), \\
\alpha=\sqrt{n_{\alpha}} e^{i \varphi_{\alpha}}, \quad \beta=\sqrt{n_{\alpha}} e^{i \varphi_{\beta}} .
\end{gathered}
$$

Получены и проанализированы СН операторов фазы ЭМ поля и числа фотонов, а также тригонометрических функций оператора фазы в состояниях (9) для любых значений $\xi$.

Константы нормировки, входящие в (9), которые могут быть найдены из условия нормировки $\left\langle\Psi_{\alpha, \beta}(\xi) \mid \Psi_{\alpha, \beta}(\xi)\right\rangle=1$, есть

$$
N_{\alpha, \beta, \xi}=1 / \sqrt{2\left(1+\cos \Theta e^{-\delta / 2}\right)},
$$

где

$$
\begin{gathered}
\delta \equiv|\alpha-\beta|^{2}=2 n_{\alpha}(1-\cos \Delta), \\
n_{\alpha}=|\alpha|^{2}, \quad n_{\beta}=n_{\alpha}, \\
\Delta \equiv \varphi_{\beta}-\varphi_{\alpha}, \\
\Theta \equiv \xi+n_{\alpha} \sin \Delta .
\end{gathered}
$$

\section{Средние значения и дисперсии операторов числа фотонов и фазы для фазовых суперпозиций когерентных состояний поля}

Среднее число фотонов для поля в состоянии фазовой суперпозиции когерентных состояний имеют вид [24]

$$
\left\langle\hat{a}^{+} \hat{a}\right\rangle=n_{\alpha} \frac{1+e^{-\delta / 2} \cos (\Delta+\Theta)}{1+e^{-\delta / 2} \cos \Theta} .
$$

Дисперсии (флуктуации) числа фотонов поля $\left\langle(\Delta n)^{2}\right\rangle \equiv\left\langle n^{2}\right\rangle-\langle n\rangle^{2}$ в состоянии фазовой суперпозиции могут быть вычислены с использованием формулы (15) для среднего числа фотонов $\langle n\rangle \equiv\left\langle a^{+} a\right\rangle$ и следующего выражения для среднего квадрата числа фотонов:

$$
\left\langle n^{2}\right\rangle=n_{0} \frac{1+n_{\alpha}+e^{-\delta / 2}\left[\cos (\Delta+\Theta)+n_{\alpha} \cos (2 \Delta+\Theta)\right]}{1+e^{-\delta / 2} \cos \Theta} .
$$

Использование приведённых формул позволяет также найти фактор Фано поля $F \equiv\left\langle(\Delta n)^{2}\right\rangle /\langle n\rangle$,

$$
\begin{aligned}
F=1 & +n_{\alpha}\left\{\frac{1+e^{-\delta / 2} \cos (2 \Delta+\Theta)}{1+e^{-\delta / 2} \cos (\Delta+\Theta)}\right. \\
& \left.-\frac{1+e^{-\delta / 2} \cos (\Delta+\Theta)}{1+e^{-\delta / 2} \cos \Theta}\right\} .
\end{aligned}
$$

Значение фактора Фано $F<1$ указывает на присутствие субпуассоновской статистики фотонов, т.е. наличие
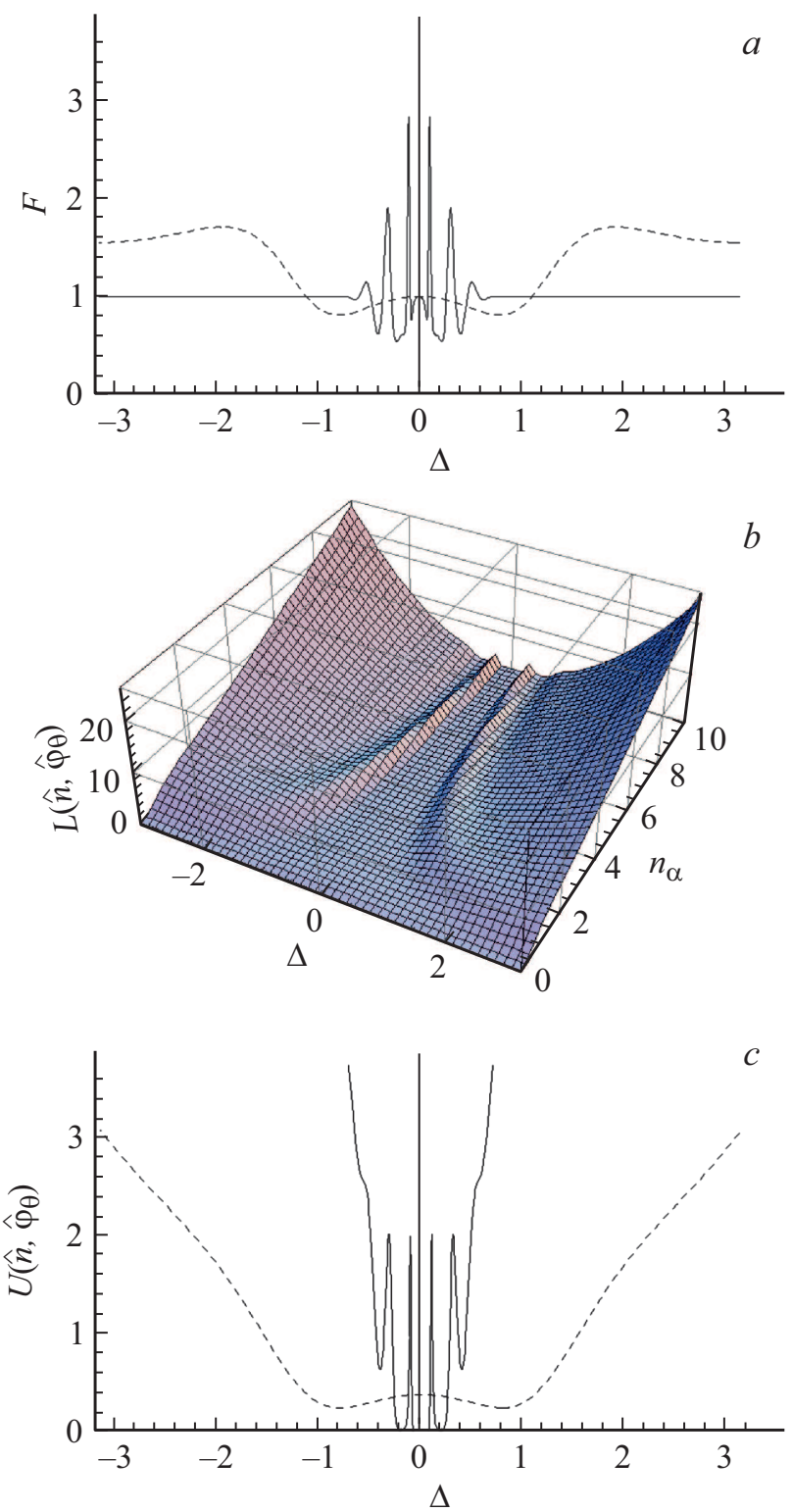

Рис. 1. (a) Зависимости фактора Фано $F=\left\langle(\Delta \hat{n})^{2}\right\rangle_{p \theta_{0, p}}$ от $\Delta=\varphi_{\beta}-\varphi_{\alpha}$ в интервале от $-\pi$ до $\pi$ для поля в состоянии фазовой суперпозиции при $n_{\alpha}=1$ (штриховая линия) и $n_{\alpha}=30$ (непрерывная линия) в случае $\xi=0$. (b) Зависимость левой части СН $L\left(\hat{n}, \hat{\varphi}_{\theta}\right) \equiv\left\langle(\Delta \hat{n})^{2}\right\rangle_{p, s}\left\langle\left(\Delta \hat{\varphi}_{\theta}\right)^{2}\right\rangle_{p, s}$ от $n_{\alpha}$ и разности фаз когерентных состояний $\Delta \equiv \varphi_{\beta}-\varphi_{\alpha}$ для поля в состоянии фазовой суперпозиции в случае $\xi=0$. (c) Зависимость разности левой и правой частей СН $U\left(\hat{n}, \hat{\varphi}_{\theta}\right) \equiv L\left(\hat{n}, \hat{\varphi}_{\theta}\right)-R\left(\hat{n}, \hat{\varphi}_{\theta}\right)$ от разности фаз когерентных состояний $\Delta \equiv \varphi_{\beta}-\varphi_{\alpha}$ для $n_{\alpha}=1$ (штриховая) и $n_{\alpha}=0$ (непрерывная линия) для поля в состоянии фазовой суперпозиции в случае $\xi=0$.

неклассического состояния поля, обладающего сжатием по числу фотонов, т. е. уровнем квантовых флуктуаций числа фотонов ниже уровня дробового шума.

На рис. 1, а приведены зависимости фактора Фано $F(\Delta)$ при $n_{\alpha}=1$ и $n_{\alpha}=30$ для $\xi=0$. Флуктуации числа фотонов для поля в фазовой суперпозиции когерентных состояний обладают сложной осциллирующей 
зависимостью от $-\pi \leq \Delta \leq \pi$, причём статистика фотонов (в зависимости от значения этой величины для фиксированных значений $\left.n_{\alpha}, \xi\right)$ может быть как супер$(F>1)$, так и субпуассоновской $(F<1)$. Зависимости $F(\Delta)$ имеют осцилляционный характер на интервале изменения $\Delta$, величина которого определяется значением величины $n_{\alpha}$. На рисунке видно, что для $n_{\alpha} \gg 1$ значение флуктуаций числа фотонов быстро осциллирует в интервале изменения $|\Delta|<0.5$, и статистика фотонов является как супер-, так субпуассоновской. Вне указанного интервала изменения $\Delta$ статистика числа фотонов пуассоновская $(F=1)$. Для $n_{\alpha} \sim 1$, как видно на рис. $1, a$ при $n_{\alpha}=1$, статистика фотонов меняется на всём интервале изменения $\Delta$ от суперпуассоновской к субпуассоновской. Особый случай представляют собой суперпозиции с $\xi \approx \pm \pi$. Для состояний суперпозиций такого вида субпуассоновская статистика числа фотонов имеет место для любых значений $\Delta$ при малых значениях $n_{\alpha}(\sim 1)$.

\section{Флуктуации и средние значения операторов фазы поля в состояниях фазовых суперпозиций когерентных состояний}

Прежде чем перейти к вычислению средних значений и дисперсий эрмитова оператора фазы (8), (7), (6) для поля в общей суперпозиции когерентных состояний найдём средние значения и дисперсии оператора фазы поля в когерентном состоянии $|\alpha\rangle$ для любых значений параметра $\theta_{0}$ :

$$
\left\langle\hat{\varphi}_{\theta}\right\rangle_{\alpha, \theta_{0}} \equiv\left\langle\alpha\left|\hat{\varphi}_{\theta}\right| \alpha\right\rangle, \quad\left\langle\hat{\varphi}_{\theta}^{2}\right\rangle_{\alpha, \theta_{0}} \equiv\left\langle\alpha\left|\hat{\varphi}_{\theta}^{2}\right| \alpha\right\rangle,
$$

где

$$
\hat{\varphi}_{\theta}^{2}=\sum_{m=0}^{S} \theta_{m}^{2}\left|\theta_{m}\right\rangle\left\langle\theta_{m}\right| .
$$

Поскольку в базисе фоковских состояний векторы когерентных состояний записываются в виде разложения

$$
|\alpha\rangle=e^{-n_{\alpha} / 2} \sum_{n=0}^{\infty} \frac{n_{\alpha}^{n / 2}}{\sqrt{n !}} e^{i n \varphi_{\alpha}}|n\rangle
$$

с помощью (20) и (7) получаем

$$
\left\langle\theta_{m} \mid \alpha\right\rangle=\frac{e^{-n_{\alpha} / 2}}{\sqrt{S+1}} \sum_{n=0}^{\infty} \frac{n_{\alpha}^{n / 2}}{\sqrt{n !}} e^{i n\left(\varphi_{\alpha}-\theta_{m}\right)}
$$

Для среднего значения $\left\langle\hat{\varphi}_{\theta}\right\rangle_{\alpha, \theta_{0}}$ находим в результате суммирования по $m$ и взятия предела $S \rightarrow \infty$ следующее выражение для среднего значения оператора фазы для когерентного состояния поля:

$$
\begin{gathered}
\left\langle\hat{\varphi}_{\theta}\right\rangle_{\alpha, \theta_{0}}=\theta_{0}+\pi+f_{1}, \\
f_{1} \equiv 2 e^{-n_{\alpha} / 2} \sum_{n>n^{\prime}}^{\infty} \frac{n_{\alpha}^{\left(n+n^{\prime}\right) / 2}}{\sqrt{n ! n^{\prime \prime}}} \frac{\sin \left[\left(n-n^{\prime}\right)\left(\theta_{0}-\varphi_{\alpha}\right)\right]}{n-n^{\prime}} .
\end{gathered}
$$

Если для произвольной величины $\theta_{0}$ в формулах (6), (22) и (23) выбрать значение

$$
\theta_{0}=\varphi_{\alpha}-\pi \frac{S}{S+1}
$$

то нетрудно убедиться, что в этом случае $f_{1}=0$ и

$$
\left\langle\hat{\varphi}_{\theta}\right\rangle_{\alpha}=\varphi_{\alpha}
$$

Используя формулы (19) и (21) для среднего квадрата фазы в когерентном состоянии поля, получаем для любого значения $\theta_{0}$ следующее:

$$
\left\langle\hat{\varphi}_{0}^{2}\right\rangle_{\alpha, \theta_{0}}=\frac{\pi^{2}}{3}+\left(\theta_{0}+\pi\right)^{2}+2 \theta_{0} f_{1}+f_{2}
$$

где

$$
f_{2} \equiv 4 e^{-n_{\alpha}} \sum_{n>n^{\prime}}^{\infty} \frac{n_{\alpha}^{\left(n+n^{\prime}\right) / 2}}{\sqrt{n ! n^{\prime} !}} \frac{\cos \left[\left(n-n^{\prime}\right)\left(\theta_{0}-\varphi_{\alpha}\right)\right]}{\left(n-n^{\prime}\right)^{2}}
$$

В свою очередь, принимая величину $\theta_{0}$ в виде (24), находим для среднего квадрата фазы формулу

$$
\left\langle\hat{\varphi}_{0}^{2}\right\rangle_{\alpha}=\frac{\pi^{2}}{3}+\varphi_{\alpha}^{2}+4 e^{-n} \sum_{n>n^{\prime}}^{\infty}(-1)^{n-n^{\prime}} \frac{n_{\alpha}^{\left(n+n^{\prime}\right) / 2}}{\sqrt{n ! n^{\prime} !}} \frac{1}{\left(n-n^{\prime}\right)^{2}},
$$

а для дисперсии фазы вследствие равенства (25) получаем следующую известную [7,17] формулу:

$$
\left\langle\left(\Delta \hat{\varphi}_{\theta}\right)^{2}\right\rangle_{\alpha}=\frac{\pi^{2}}{3}+4 e^{-n} \sum_{n>n^{\prime}}^{\infty}(-1)^{n-n^{\prime}} \frac{n_{\alpha}^{\left(n+n^{\prime}\right) / 2}}{\sqrt{n ! n^{\prime} !}} \frac{1}{\left(n-n^{\prime}\right)^{2}} .
$$

Перейдем далее к расчету средних значений и дисперсий фазы поля в состоянии общей суперпозиции когерентных состояний вида

$$
\begin{gathered}
\left|\Psi_{\alpha, \beta}(\xi)\right\rangle=N_{\alpha, \beta, \xi}\left(|\alpha\rangle+e^{i \xi}|\beta\rangle\right), \\
\alpha=\sqrt{n_{\alpha}} e^{i \varphi_{\alpha}}, \quad \beta=\sqrt{n_{\beta}} e^{i \varphi_{\beta}} .
\end{gathered}
$$

Входящий в (30) нормировочный фактор в данном случае равен

$$
N_{\alpha, \beta, \xi}=\frac{1}{\sqrt{2\left(1+\cos \Theta e^{-\delta / 2}\right)}},
$$

где введены обозначения $\delta \equiv|\alpha-\beta|^{2}=n_{\alpha}+n_{\beta}$ $-2 \sqrt{n_{\alpha} n_{\beta}} \cos \Delta, \Delta \equiv \varphi_{\beta}-\varphi_{\alpha}, \Theta \equiv n_{\alpha} \sin \Delta+\xi$.

Средние и дисперсии эрмитова оператора фазы поля, определяемого согласно (8)-(6), для поля в общей суперпозиции когерентных состояний $(30)$ и произвольной $\theta_{0}$ есть

$$
\begin{aligned}
\left\langle\hat{\varphi}_{\theta}\right\rangle_{\alpha, \beta, \theta_{0}} & \equiv\left\langle\Psi_{\alpha, \beta}(\xi)\left|\hat{\varphi}_{\theta}\right| \Psi_{\alpha, \beta}(\xi)\right\rangle, \\
\left\langle\hat{\varphi}_{\theta}^{2}\right\rangle_{\alpha, \beta, \theta_{0}} & \equiv\left\langle\Psi_{\alpha, \beta}(\xi)\left|\hat{\varphi}_{\theta}^{2}\right| \Psi_{\alpha, \beta}(\xi)\right\rangle .
\end{aligned}
$$

Подставляя (8), (30) и (21) в первое из равенств (32) и используя следующее из (7), (20) соотношение (21), выполняя суммирование по $m$, после взятия предела 
$m \rightarrow \infty$ находим для среднего фазы поля в состоянии общей суперпозиции следующее выражение:

$$
\begin{aligned}
\left\langle\hat{\varphi}_{\theta}\right\rangle_{\alpha, \beta, \theta_{0}}= & N_{\alpha, \beta, \xi}^{2}\left\{\left\langle\hat{\varphi}_{\theta}\right\rangle_{\alpha, \theta_{0}}+\left\langle\hat{\varphi}_{\theta}\right\rangle_{\beta, \theta_{0}}\right. \\
& \left.+2 e^{\frac{n_{\alpha}+n_{\beta}}{2}}\left[S_{0}\left(\theta_{0}+\pi\right)+S_{1}\right]\right\},
\end{aligned}
$$

где

$$
\begin{aligned}
& S_{0} \equiv \sum_{n=0}^{\infty} \frac{n_{\alpha}^{n / 2} n_{\beta}^{n / 2}}{n !} \cos [\Delta n+\xi]=e^{\sqrt{n_{\alpha} n_{\beta}} \cos \Delta} \\
& \times \cos \left[\sqrt{n_{\alpha} n_{\beta}} \sin \Delta+\xi\right], \\
& S_{1} \equiv \sum_{n \neq n^{\prime}}^{\infty} \frac{n_{\alpha}^{n / 2} n_{\beta}^{n^{\prime} / 2}}{\sqrt{n ! n^{\prime \prime}}} \frac{\sin \left[\varphi_{\beta} n^{\prime}-\varphi_{\alpha} n+\theta_{0}\left(n-n^{\prime}\right)+\xi\right]}{n-n^{\prime}} .
\end{aligned}
$$

Величины $\left\langle\hat{\varphi}_{\theta}\right\rangle_{\alpha, \theta_{0}}$ и $\left\langle\hat{\varphi}_{\theta}\right\rangle_{\beta, \theta_{0}}$, входящие в (33), представляют собой средние значения фазы поля в когерентном состоянии для произвольного значения $\theta_{0}$. Для них нами выше получены выражения (22), (23). Среднее квадрата оператора фазы (19) в состояние фазовой суперпозиции (30) для произвольного значения параметра $\theta_{0}$ также находится путем суммирования по $m$ и взятия предела $S \rightarrow \infty$ и имеет следующий вид:

$$
\begin{aligned}
& \left\langle\hat{\varphi}_{\theta}^{2}\right\rangle_{\alpha, \beta, \theta_{0}}=N_{\alpha, \beta, \xi}^{2}\left\{\left\langle\hat{\varphi}_{\theta}^{2}\right\rangle_{\alpha, \theta_{0}}+\left\langle\hat{\varphi}_{\theta}^{2}\right\rangle_{\beta, \theta_{0}}+2 e^{\frac{n_{\alpha}+n_{\beta}}{2}}\right. \\
& \left.\times\left\{\left[\frac{4}{3} \pi^{2}+\theta_{0}\left(\theta_{0}+2 \pi\right)\right] S_{0}+2 \theta_{0} S_{1}+2 S_{2}\right\}\right\}, \\
& S_{2} \equiv \sum_{n \neq n^{\prime}}^{\infty} \frac{n_{\alpha}^{n / 2} n_{\beta}^{n^{\prime} / 2}}{\sqrt{n ! n^{\prime} !}} \frac{\cos \left[\varphi_{\beta} n^{\prime}-\varphi_{\alpha} n+\theta_{0}\left(n-n^{\prime}\right)+\xi\right]}{\left(n-n^{\prime}\right)^{2}} .
\end{aligned}
$$

Величины $\left\langle\hat{\varphi}_{\theta}^{2}\right\rangle_{\alpha, \theta_{0}}$ и $\left\langle\hat{\varphi}_{\theta}^{2}\right\rangle_{\beta, \theta_{0}}$, входящие в (28), представляют собой средние значения квадрата фазы поля в когерентном состоянии для произвольного значения $\theta_{0}$. Для них нами выше получено выражение (26). В частном случае фазовых суперпозиций в формулах (33)-(37) полагается $n_{\alpha}=n_{\beta}$, а для $\theta_{0}$ выбирается значение, соответствующее данному квантовому состоянию.

Для расчетов средних значений и дисперсий фазы поля в состояниях фазовых суперпозиций когерентных состояний (9) нами используется следующее значение параметра $\theta_{0}$, определяющего дискретный базис собственных функций оператора $\hat{\varphi}_{\theta}$, соответствующий рассматриваемым нами квантовым состояниям поля:

$$
\theta_{0, p}=\frac{\varphi_{\alpha}+\varphi_{\beta}}{2}-\pi \frac{S}{S+1} .
$$

Подстановка (38) в общие выражения для среднего значения фазы (33)-(35) для поля в состоянии (9) позволяет получить для этой величины формулу

$$
\left\langle\hat{\varphi}_{\theta}\right\rangle_{\text {p.s. }}=\frac{\varphi_{\alpha}+\varphi_{\beta}}{2} .
$$

Из (36), (37) и (33) с учетом (39) находим для дисперсии фазы поля

$$
\left\langle\left(\Delta \hat{\varphi}_{\theta}\right)^{2}\right\rangle_{\text {p.s. }} \equiv\left\langle\hat{\varphi}_{\theta}^{2}\right\rangle_{\text {p.s. }}-\left\langle\hat{\varphi}_{\theta}\right\rangle_{\text {p.s. }}^{2}
$$

в фазовой суперпозиции когерентных состояний (9) выражение [25]

$$
\begin{gathered}
\left\langle\left(\Delta \hat{\varphi}_{\theta}\right)^{2}\right\rangle_{\text {p.s. }}=\frac{\pi^{2}}{3}+\frac{8 e^{-n_{\alpha}}}{1+e^{-2 n_{\alpha} \sin ^{2} \frac{\Delta}{2}} \cos \left(n_{\alpha} \sin \Delta+\xi\right)} \\
\times \sum_{n>n^{\prime}}^{\infty}(-1)^{n-n^{\prime}} \frac{n_{\alpha}^{\left(n+n^{\prime}\right) / 2}}{\sqrt{n ! n^{\prime} !}} \frac{\cos \left(\frac{n \Delta+\xi}{2}\right) \cos \left(\frac{n^{\prime} \Delta+\xi}{2}\right)}{\left(n-n^{\prime}\right)^{2}},
\end{gathered}
$$

где $\Delta \equiv \varphi_{\beta}-\varphi_{\alpha}$.

Из формулы (40) следует, что флуктуации фазы поля в состояниях фазовых суперпозиций зависят не от отдельных значений фаз когерентных состояний, составляющих суперпозицию, а от разности этих фаз $\Delta \equiv \varphi_{\beta}-\varphi_{\alpha}$

Расчеты показали, что с ростом $n_{\alpha}$ при уменьшении абсолютной величины разности фазовых углов когерентных состояний $|\Delta|$ в интервале изменения $\Delta$ от $\pi$ до $-\pi$ для любых значений $\xi$ величина флуктуаций (дисперсий) фазы стремится к 0 , а при увеличении $|\Delta|$ флуктуации фазы возрастают. При $n_{\alpha} \gg 1$ для дисперсии фазы поля в общем фазовом состоянии выполняется приближенное равенство

$$
\left\langle\left(\Delta \hat{\varphi}_{\theta}\right)^{2}\right\rangle_{\text {p.s. }} \approx \frac{\Delta^{2}}{4}
$$

Формула (41) означает, что для поля как в мезо-, так и в макроскопическом состоянии (для сколь угодно большого значения среднего числа фотонов) флуктуации фазы поля в общей фазовой суперпозиции когерентных состояний велики при больших разностях фазовых углов когерентных состояний $|\Delta|$.

Расчеты также показывают, что наибольшими квантовыми флуктуациями фазы обладают фазовые суперпозиции когерентных состояний поля при $|\Delta|=\pi$ для любых значений $n_{\alpha}>0$ и $\xi$, т.е. состояния „шредингеровского кота“" вида

$$
\left|\Psi_{\alpha,-\alpha}(\xi)\right\rangle=N_{\alpha,-\alpha, \xi}\left(|\alpha\rangle+e^{i \xi}|-\alpha\rangle\right)
$$

Отметим, что в случае когерентного состояния поля (т.е. при $\Delta=0$ ) флуктуации фазы поля в мезо- и макроскопических состояниях $\left(n_{\alpha} \gg 1\right)$ близки к нулю.

\section{Корреляции операторов фазы и числа фотонов и СН число фотонов-фаза поля в состояниях фазовых суперпозиций когерентных состояний}

Используя равенства (8) и (7), (6), для оператора произведения операторов числа фотонов и фазы можно получить

$$
\hat{n} \hat{\varphi}_{\theta}=\theta_{0} \hat{n}+\frac{2 \pi}{(S+1)^{2}} \sum_{m=0}^{S} \sum_{n, n^{\prime}=0}^{\infty} e^{i\left(n-n^{\prime}\right) \theta_{m}} n m|n\rangle\left\langle n^{\prime}\right|,
$$




$$
\theta_{0}=\frac{\varphi_{\alpha}+\varphi_{\beta}}{2}-\pi
$$

поскольку

$$
\begin{gathered}
\hat{\varphi}_{\theta}=\theta_{0}+\frac{2 \pi}{S+1} \sum_{m=0}^{S} m\left|\theta_{m}\right\rangle\left\langle\theta_{m}\right|, \\
\hat{n}=\sum_{n=0}^{\infty} n|n\rangle\langle n| .
\end{gathered}
$$

Проводя суммирование по $m$ от 0 до $S \gg 1$ в (42), находим

$$
\hat{n} \hat{\varphi}_{\theta} \approx\left(\theta_{0}+\pi\right)+\sum_{n \neq n^{\prime}}^{\infty} e^{i\left(n-n^{\prime}\right) \theta_{m}} \frac{n}{i\left(n-n^{\prime}\right)}|n\rangle\left\langle n^{\prime}\right| .
$$

Выполнив расчет квантово-механического среднего $\langle\ldots\rangle_{\text {p.s. }}$ от оператора (43) в состояниях суперпозиций (9), получаем

$$
\begin{aligned}
& \left\langle\hat{n} \hat{\varphi}_{\theta}\right\rangle_{\text {p.s. }}=\left\langle\hat{\varphi}_{\theta}\right\rangle_{\text {p.s. }}\langle\hat{n}\rangle_{\text {p.s. }}+2 N_{\alpha \beta \xi}^{2} e^{-n_{\alpha}} \sum_{n \neq n^{\prime}}^{\infty}(-1)^{n-n^{\prime}} \frac{n_{\alpha}^{\frac{n+n^{\prime}}{2}}}{\sqrt{n ! n^{\prime} !}} \\
& \times \frac{n}{i\left(n-n^{\prime}\right)}\left\{\cos \left[\frac{\Delta}{2}\left(n-n^{\prime}\right)\right]+\cos \left[\frac{\Delta}{2}\left(n+n^{\prime}\right)+\xi\right]\right\} .
\end{aligned}
$$

Проведя подобные расчеты для $\left\langle\hat{\varphi}_{\theta} \hat{n}\right\rangle_{\text {p.s. }}$, находим следующее выражение для среднего коммутатора числа фотонов и фазы:

$$
\begin{aligned}
& \left\langle\left[\hat{n}, \hat{\varphi}_{\theta}\right]\right\rangle_{\text {p.s. }}=2 N_{\alpha \beta \xi}^{2} e^{-n_{\alpha}} \sum_{n \neq n^{\prime}}^{\infty}(-1)^{n-n^{\prime}} \frac{n_{\alpha}^{\frac{n+n^{\prime}}{2}}}{i \sqrt{n ! n^{\prime} !}} \\
& \times\left\{\cos \left[\frac{\Delta}{2}\left(n-n^{\prime}\right)\right]+\cos \left[\frac{\Delta}{2}\left(n+n^{\prime}\right)+\xi\right]\right\} .
\end{aligned}
$$

Сравнивая (44) с (45), нетрудно убедиться, что

$$
\left|\left\langle\left[\hat{n}, \hat{\varphi}_{\theta}\right]\right\rangle_{\text {p.s. }}\right|=2\left|\left\langle\hat{n}, \hat{\varphi}_{\theta}\right\rangle_{\text {p.s. }}\right| .
$$

Таким образом, показано, что правые части жесткого $\mathrm{CH}($ ЖН $)$ (5) и мягкого $\mathrm{CH}(\mathrm{MCH})(4)$ при $\hat{A}=\hat{n}$, $\hat{B}=\hat{\varphi}_{\theta}$ равны между собой в рассматриваемом нами случае операторов числа фотонов и фазы поля для фазовой суперпозиции когерентных состояний.

Из полученного нами выражения (44) следует, что корреляции числа фотонов и фазы зависят только от разности фаз когерентных состояний $\Delta \equiv \varphi_{\beta}-\varphi_{\alpha}$. Формулы (17) и (40) для дисперсий числа фотонов и фазы поля также указывают на зависимости этих величин только от $\Delta$. Как следует из выражения (46), правые части ЖСН и МСН определяются с помощью корреляций (44) согласно

$$
\begin{aligned}
& \left|\left\langle\hat{n}, \hat{\varphi}_{\theta}\right\rangle_{\text {p.s. }}\right|^{2}=\frac{\left|\left\langle\left[\hat{n}, \hat{\varphi}_{\theta}\right]\right\rangle_{\text {p.s. }}\right|^{2}}{4}=4 \mid N_{\alpha \beta \xi}^{2} e^{-n_{\alpha}} \sum_{n \neq n^{\prime}}^{\infty} \frac{n_{\alpha}^{\frac{n+n^{\prime}}{2}}}{i \sqrt{n ! n^{\prime} !}} \\
& \times\left.(-1)^{n-n^{\prime}}\left\{\cos \left[\frac{\Delta}{2}\left(n-n^{\prime}\right)\right]+\cos \left[\frac{\Delta}{2}\left(n+n^{\prime}\right)+\xi\right]\right\}\right|^{2} .
\end{aligned}
$$

Флуктуации фазы $\left\langle\left(\Delta \hat{\varphi}_{\theta}\right)^{2}\right\rangle_{\text {p.s. }}$, входящие в левую часть $\mathrm{CH}$, при $n_{\alpha} \gg 1$ имеют зависимость (41) от разности фаз когерентных состояний, это означает, что флуктуации фазы значительны для мезо- и макроскопических полей с большими $n_{\alpha}$ в случае больших значений $|\Delta|$ и не убывают с ростом среднего числа фотонов. Флуктуации же числа фотонов поля в рассматриваемом нами состоянии увеличиваются с ростом среднего числа фотонов.

Пример зависимости левой части $\mathrm{CH}$ $L\left(\hat{n}, \hat{\varphi}_{\theta}\right) \equiv\left\langle(\Delta \hat{n})^{2}\right\rangle_{\text {p.s. }}\left\langle\left(\Delta \hat{\varphi}_{\theta}\right)^{2}\right\rangle_{\text {p.s. }} \quad$ от $\quad \Delta \quad$ приведен $\quad$ на рис. $1, b$. На рисунке показано, что левая часть $\mathrm{CH}$ близка к $1 / 4$ в узком интервале изменения $|\Delta| \approx 0$ и резко возрастает для больших $|\Delta|$ в случае $n_{\alpha} \gg 1$. С ростом $n_{\alpha}$ при $|\Delta| \approx \pi$, т.е. в случае макроскопических состояний нечётных суперпозиций когерентных состояний, левые части СН неограниченно возрастают линейно по $n_{\alpha} \gg 1$.

Как отмечалось выше, правые части МСН и ЖСН числа фотонов-фазы поля в случае фазовых суперпозиций когерентных состояний совпадают между собой для любых $n_{\alpha}$ и $\xi$. То есть

$$
\begin{gathered}
R\left(\hat{n}, \hat{\varphi}_{\theta}\right) \equiv R_{t}\left(\hat{n}, \hat{\varphi}_{\theta}\right)=R_{s}\left(\hat{n}, \hat{\varphi}_{\theta}\right), \\
R_{t}\left(\hat{n}, \hat{\varphi}_{\theta}\right) \equiv\left|\left\langle\hat{n}, \hat{\varphi}_{\theta}\right\rangle_{\text {p.s. }}\right|^{2}, \\
R_{s}\left(\hat{n}, \hat{\varphi}_{\theta}\right) \equiv \frac{1}{4}\left|\left\langle\left[\hat{n}, \hat{\varphi}_{\theta}\right]\right\rangle_{\text {p.s. }}\right|^{2} .
\end{gathered}
$$

Расчеты показали, что $L\left(\hat{n}, \hat{\varphi}_{\theta}\right)-R\left(\hat{n}, \hat{\varphi}_{\theta}\right) \approx 0$ только в узких диапазонах изменения $\Delta$ вблизи $|\Delta|=0$ и $n_{\alpha} \gg 1$, как показано на рис. 1,c. Это означает, что состояние поля фазовой суперпозиции когерентных состояний не является в общем случае состоянием с минимальной неопределённостью число фотонов-фаза поля.

\section{СН для числа фотонов и тригонометрических функций операторов фазы поля в состояниях фазовых суперпозиций когерентных состояний}

Рассмотрим теперь тригонометрические функции от эрмитова оператора фазы $\hat{\varphi}_{\theta}$, определяемого в $(S+1)$ мерном пространстве фоковских состояний,

$$
\cos \hat{\varphi_{\theta}}=\frac{e^{i \hat{\varphi}_{\theta}}+e^{-i \hat{\varphi}_{\theta}}}{2}, \quad \sin \hat{\varphi_{\theta}}=\frac{e^{i \hat{\varphi}_{\theta}}-e^{-i \hat{\varphi}_{\theta}}}{2 i} .
$$


Операторные функции $e^{i \hat{\varphi}_{\theta}}$ и $e^{-i \hat{\varphi}_{\theta}}$, входящие в (48), унитарны, поскольку оператор фазы $\hat{\varphi}_{\theta}$ эрмитов.

Используя соотношения

$$
\begin{gathered}
|n\rangle=\frac{1}{\sqrt{S+1}} \sum_{m=0}^{S} e^{-i n \theta_{m}}\left|\theta_{m}\right\rangle, \\
e^{i \hat{\varphi}_{\theta}}|n\rangle=\frac{1}{\sqrt{S+1}} \sum_{m=0}^{S} e^{i(n-1) \theta_{m}}\left|\theta_{m}\right\rangle,
\end{gathered}
$$

может быть показано [7] , что

$$
\begin{aligned}
e^{i \hat{\varphi}_{\theta}} & =\sum_{n=1}^{S}|n-1\rangle\left\langle n\left|+e^{i(S+1) \theta_{0}}\right| S\right\rangle\langle 0|, \\
e^{-i \hat{\varphi}_{\theta}} & =\sum_{n=1}^{S}|n\rangle\left\langle n-1\left|+e^{-i(S+1) \theta_{0}}\right| 0\right\rangle\langle S| .
\end{aligned}
$$

Общее состояние суперпозиции когерентных состояний поля (30) может быть представлено в виде разложения в базисе фоковских состояний как

$$
\left|\Psi_{\alpha, \beta}(\xi)\right\rangle=\sum_{m=0}^{\infty} Z_{m}(\alpha, \beta, \xi)|m\rangle,
$$

где обозначено

$$
Z_{m}(\alpha, \beta, \xi) \equiv N_{\alpha, \beta, \xi}\left(\alpha^{m} e^{-n_{\alpha} / 2}+e^{i \xi} \beta^{m} e^{-n_{\beta} / 2}\right) / \sqrt{m !} .
$$

Используя (51)-(54), для средних значений периодических тригонометрических функций оператора фазы (48) поля в состоянии общей суперпозиции когерентных состояний можно найти следующие выражения:

$$
\begin{aligned}
\left\langle\cos \hat{\varphi}_{\theta}\right\rangle_{\text {p.s. }}= & \lim _{S \rightarrow \infty} \operatorname{Re}\left[\sum_{n=0}^{S-1}(\alpha, \beta, \xi) Z_{n+1}(\alpha, \beta, \xi)\right. \\
& \left.+e^{i(S+1) \theta_{0}} Z_{S}^{*}(\alpha, \beta, \xi) Z_{0}(\alpha, \beta, \xi)\right], \\
\left\langle\sin \hat{\varphi}_{\theta}\right\rangle_{\text {p.s. }}= & \lim _{S \rightarrow \infty} \operatorname{Re}\left[\sum_{n=0}^{S-1}(\alpha, \beta, \xi) Z_{n+1}(\alpha, \beta, \xi)\right. \\
& \left.+e^{i(S+1) \theta_{0}} Z_{S}^{*}(\alpha, \beta, \xi) Z_{0}(\alpha, \beta, \xi)\right],
\end{aligned}
$$

Вторые слагаемые в квадратных скобках в формулах (55) и (56) стремятся к 0 при $S \rightarrow \infty$, поскольку $n_{\alpha}^{S / 2} e^{-n_{\alpha} / 2} / \sqrt{S !}, n_{\beta}^{S / 2} e^{-n_{\beta} / 2} / \sqrt{S !} \rightarrow 0$ при $S \rightarrow \infty$. Вследствие этого средние значения тригонометрических функций оператора фазы (в отличие от средних значений самого оператора фазы $\hat{\varphi}_{\theta}$ ) оказываются не зависящими от параметра $\theta_{0}$.
Для дисперсий тригонометрических функций эрмитова оператора фазы в состоянии фазовой суперпозиции (9) находим

$$
\begin{aligned}
& \left\langle\left(\Delta \cos \hat{\varphi}_{\theta}\right)^{2}\right\rangle_{\text {p.s. }}=\frac{1}{2}+\frac{1}{2} \sum_{n=0}^{\infty} \operatorname{Re}\left[Z_{n}^{*}(\alpha, \beta, \xi) Z_{n+2}(\alpha, \beta, \xi)\right] \\
& -\left(\sum_{n=0}^{\infty} \operatorname{Re}\left[Z_{n}^{*}(\alpha, \beta, \xi) Z_{n+1}(\alpha, \beta, \xi)\right]\right)^{2}, \\
& \left\langle\left(\Delta \sin \hat{\varphi}_{\theta}\right)^{2}\right\rangle_{\text {p.s. }}=\frac{1}{2}-\frac{1}{2} \sum_{n=0}^{\infty} \operatorname{Re}\left[Z_{n}^{*}(\alpha, \beta, \xi) Z_{n+2}(\alpha, \beta, \xi)\right] \\
& \quad-\left(\sum_{n=0}^{\infty} \operatorname{Im}\left[Z_{n}^{*}(\alpha, \beta, \xi) Z_{n+1}(\alpha, \beta, \xi)\right]\right)^{2} .
\end{aligned}
$$

Из формул (57) и (58) следует, что для рассматриваемых нами состояний поля дисперсии периодических тригонометрических функций оператора фазы не зависят от параметра $\theta_{0}$.

Численные расчеты показывают, что для дисперсий косинуса и синуса фазы поля в состояниях фазовых суперпозиций при $n_{\alpha} \gg 1$ справедливы соотношения [24]

$$
\begin{aligned}
\left\langle\left(\cos \hat{\varphi}_{\theta}\right\rangle_{\text {p.s. }}\right. & \approx \frac{\cos \varphi_{\alpha}+\cos \varphi_{\beta}}{2}, \\
\left\langle\left(\sin \hat{\varphi}_{\theta}\right\rangle_{\text {p.s. }}\right. & \approx \frac{\sin \varphi_{\alpha}+\sin \varphi_{\beta}}{2}, \\
\left\langle\left(\cos ^{2} \hat{\varphi}_{\theta}\right\rangle_{\text {p.s. }}\right. & \approx \frac{\cos ^{2} \varphi_{\alpha}+\cos ^{2} \varphi_{\beta}}{2}, \\
\left\langle\left(\sin ^{2} \hat{\varphi}_{\theta}\right\rangle_{\text {p.s. }}\right. & \approx \frac{\sin ^{2} \varphi_{\alpha}+\sin ^{2} \varphi_{\beta}}{2}, \\
\left\langle\left(\Delta \cos \hat{\varphi}_{\theta}\right)^{2}\right\rangle_{\text {p.s. }} & \approx \frac{\left(\cos \varphi_{\alpha}-\cos \varphi_{\beta}\right)^{2}}{4}, \\
\left\langle\left(\Delta \sin \hat{\varphi}_{\theta}\right)^{2}\right\rangle_{\text {p.s. }} & \approx \frac{\left(\sin \varphi_{\alpha}-\sin \varphi_{\beta}\right)^{2}}{4} .
\end{aligned}
$$

Используя формулы (48), (51) и (52), получаем

$$
\begin{aligned}
& \hat{n} \cos \hat{\varphi}_{\theta}=\frac{1}{2} \sum_{n=0}^{S-1} n|n\rangle\langle n+1|+(n+1)| n+1\rangle\langle n| \\
& +e^{i(S+1) \theta_{0, p}} S|S\rangle\langle 0|, \quad \theta_{0, p}=\frac{\varphi_{\alpha}+\varphi_{\beta}}{2}-\pi .
\end{aligned}
$$

С помощью формул (53) и (54) находим среднее квантово-механическое оператора (62) в состоянии фазовой суперпозиции

$$
\begin{aligned}
\left\langle\hat{n} \cos \hat{\varphi}_{\theta}\right\rangle_{\text {p.s. }}=\sum_{n=0}^{\infty} n \operatorname{Re}\left[Z_{n}^{*}(\alpha, \beta, \xi) Z_{n+1}(\alpha, \beta, \xi)\right] \\
+\frac{1}{2} Z_{n+1}^{*}(\alpha, \beta, \xi) Z_{n}(\alpha, \beta, \xi),
\end{aligned}
$$




$$
\begin{aligned}
\left\langle\cos \hat{\varphi}_{\theta} \hat{n}\right\rangle_{\text {p.s. }}= & \sum_{n=0}^{\infty} n \operatorname{Re}\left[Z_{n}^{*}(\alpha, \beta, \xi) Z_{n+1}(\alpha, \beta, \xi)\right] \\
& +\frac{1}{2} Z_{n}^{*}(\alpha, \beta, \xi) Z_{n+1}(\alpha, \beta, \xi) .
\end{aligned}
$$

Для получения соотношения (63) использовалось $S Z_{S}^{*}(\alpha, \beta, \xi) \rightarrow 0$ при $S \rightarrow \infty, \forall n_{\alpha} \gg 1$.

Отметим, что корреляции (63) определяют правую часть ЖСН согласно (5) для $\hat{A}=\hat{n}, \hat{B}=\cos \hat{\varphi}_{\theta}$. Правая часть МСН определяет среднее квантово-механическое от коммутатора операторов числа фотонов и косинуса оператора фазы поля. Из соотношений (63a) и (63b) следует, что среднее такого коммутатора есть

$$
\left\langle\left[\hat{n}, \cos \hat{\varphi}_{\theta}\right]\right\rangle_{\text {p.s. }}=i \sum_{n=0}^{\infty} \operatorname{Im}\left[Z_{n+1}^{*}(\alpha, \beta, \xi) Z_{n}(\alpha, \beta, \xi)\right] .
$$

Флуктуации (дисперсии) косинуса оператора фазы

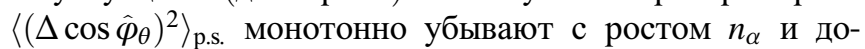
стигают величины $\approx 0$ для $|\Delta|$ вблизи 0 при $n_{\alpha} \gg 1$. Для больших $n_{\alpha}$ с увеличением $|\Delta|$ флуктуации косинуса фазы монотонно возрастают и достигают величины $\approx 1$ при $|\Delta| \approx \pi$.

Левая часть $\mathrm{CH} L\left(\hat{n}, \cos \hat{\varphi}_{\theta}\right) \equiv\left\langle(\Delta \hat{n})^{2}\right\rangle_{\text {p.s. }}\left\langle\left(\Delta \cos \hat{\varphi}_{\theta}\right)^{2}\right\rangle_{\text {p.s. }}$ обладает зависимостью от $n_{\alpha}$ и $\Delta, \quad$ подобной $L\left(\hat{n}, \hat{\varphi}_{\theta}\right) \equiv\left\langle(\Delta \hat{n})^{2}\right\rangle_{\text {p.s. }}\left\langle\left(\Delta \hat{\varphi}_{\theta}\right)^{2}\right\rangle_{\text {p.s. }} \quad$ и $\quad$ изображенной $\quad$ на рис. $1, b$ при тех же значениях параметров, но достигает значительно меньшей максимальной величины $\approx n_{\alpha}$ при $n_{\alpha} \gg 1$ и $|\Delta| \approx \pi$.

На рис. $2, a$ показан пример зависимости правой части ЖСН $R_{t}\left(\hat{n}, \cos \hat{\varphi}_{\theta}\right)$ от $n_{\alpha}$ и $\Delta$. Как видно на рисунке, правая часть ЖСН близка к нулю $(<0.06)$ для всех значений параметров $n_{\alpha}$ и $\Delta$, за исключением малой области $n_{\alpha} \approx 1.5$ и $\Delta \approx 1.2$, где присутствует ярко выраженный пик значения $R_{t}\left(\hat{n}, \cos \hat{\varphi}_{\theta}\right) \approx 0.2$.

В отличие от $\mathrm{CH}$ для числа фотонов-оператора фазы, рассмотренного нами выше, правые части ЖСН и $\mathrm{MCH}$ для числа фотонов-косинуса оператора фазы различаются между собой. На рис. $2, b$ показана зависимость разности правых частей ЖСН и $\mathrm{MCH}$ $R_{t}\left(\hat{n}, \cos \hat{\varphi}_{\theta}\right)-R_{s}\left(\hat{n}, \cos \hat{\varphi}_{\theta}\right)$ от $n_{\alpha}$ и $\Delta$ для значений параметров, использованных для рис. $2, a$. На рисунке видно, что правые части $\mathrm{MCH}$ и ЖСН совпадают между собой для $n_{\alpha} \gg 1$ и заметно различаются при $n_{\alpha} \sim 1$ в областях максимальных значений правой части ЖСН.

Разность левой и правой частей ЖСН и $\mathrm{MCH}$ $U_{x}\left(\hat{n}, \cos \hat{\varphi}_{\theta}\right) \equiv L\left(\hat{n}, \cos \hat{\varphi}_{\theta}\right)-R_{x}\left(\hat{n}, \cos \hat{\varphi}_{\theta}\right), x \equiv t, s$ близка к 0 в ограниченном интервале вблизи $|\Delta|=0$ для $n_{\alpha} \gg 1$ и велика $\left(\approx n_{\alpha}\right)$ при значениях $n_{\alpha} \gg 1$. На рис. $2, c$ изображены зависимости $U_{t}\left(\hat{n}, \cos \hat{\varphi}_{\theta}\right)$ от $\Delta$ для большого и малого значений $n_{\alpha}$. Как показано на рисунке, $U_{t}\left(\hat{n}, \cos \hat{\varphi}_{\theta}\right)$ для больших $n_{\alpha}$ близка к 0 в интервале значений разности фаз $|\Delta|<0.4$ и быстро растет с увеличением $|\Delta|$ вне этого интервала. Для малых $n_{\alpha}$ разность левой и правой частей ЖСН $U_{t}\left(\hat{n}, \cos \hat{\varphi}_{\theta}\right)$ близка к 0 на более широком интервале изменения $\Delta$.
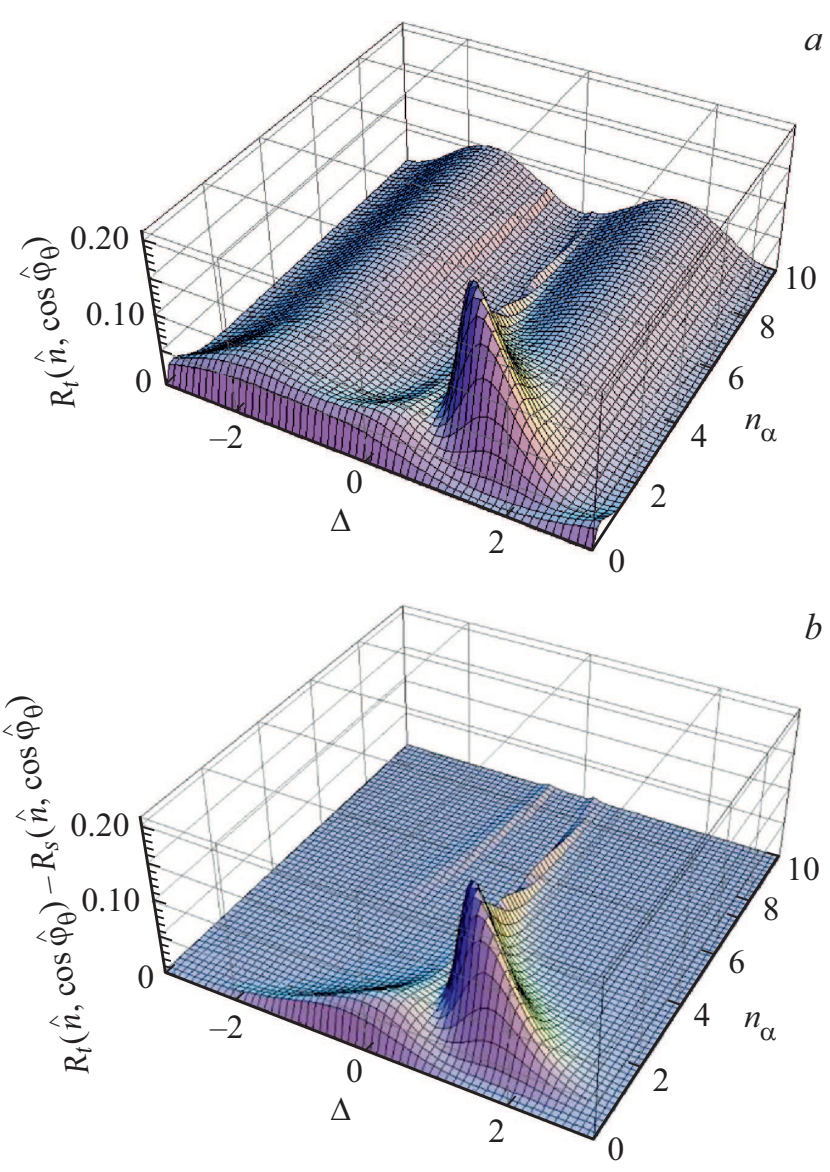

$b$

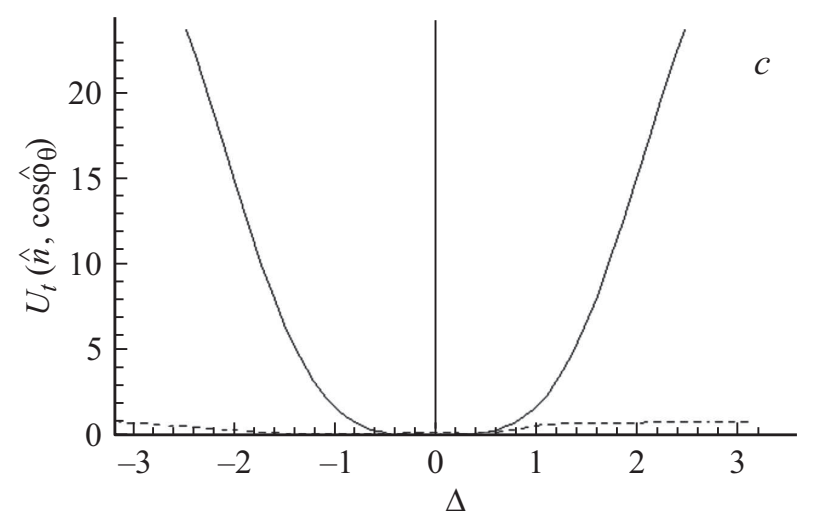

Рис. 2. (a) Зависимость правой части жесткого $\mathrm{CH}$ $R_{t}\left(\hat{n}, \cos \hat{\varphi}_{\theta}\right) \equiv\left|\left\langle\hat{n}, \cos \hat{\varphi}_{\theta}\right\rangle_{p, s}\right|^{2} \quad$ от $n_{\alpha} \quad$ и разности фаз когерентных состояний $\Delta \equiv \varphi_{\beta}-\varphi_{\alpha}$ для поля в состоянии фазовой суперпозиции вида в случае $\xi=\pi / 2, \varphi_{\alpha}=0$. (b) Зависимость разности правых частей ЖСН и $\mathrm{MCH}$ $R_{t}\left(\hat{n}, \cos \hat{\varphi}_{\theta}\right)-R_{s}\left(\hat{n}, \cos \hat{\varphi}_{\theta}\right) \quad$ от $\quad n_{\alpha} \quad$ и $\quad$ разности фаз когерентных состояний $\Delta \equiv \varphi_{\beta}-\varphi_{\alpha}$ для поля в состоянии фазовой суперпозиции в случае $\xi=\pi / 2, \varphi_{\alpha}=0$. (c) Зависимость разности левой и правой частей ЖСН $U_{t}\left(\hat{n}, \cos \hat{\varphi}_{\theta}\right) \equiv L\left(\hat{n}, \cos \hat{\varphi}_{\theta}\right)-R_{t}\left(\hat{n}, \cos \hat{\varphi}_{\theta}\right)$ от разности фаз когерентных состояний $\Delta \equiv \varphi_{\beta}-\varphi_{\alpha}$ для $n_{\alpha}=1$ (штрихи) и $n_{\alpha}=30$ (непрерывная линия) для поля в состоянии фазовой суперпозиции в случае $\xi=0, \varphi_{\alpha}=0$. 

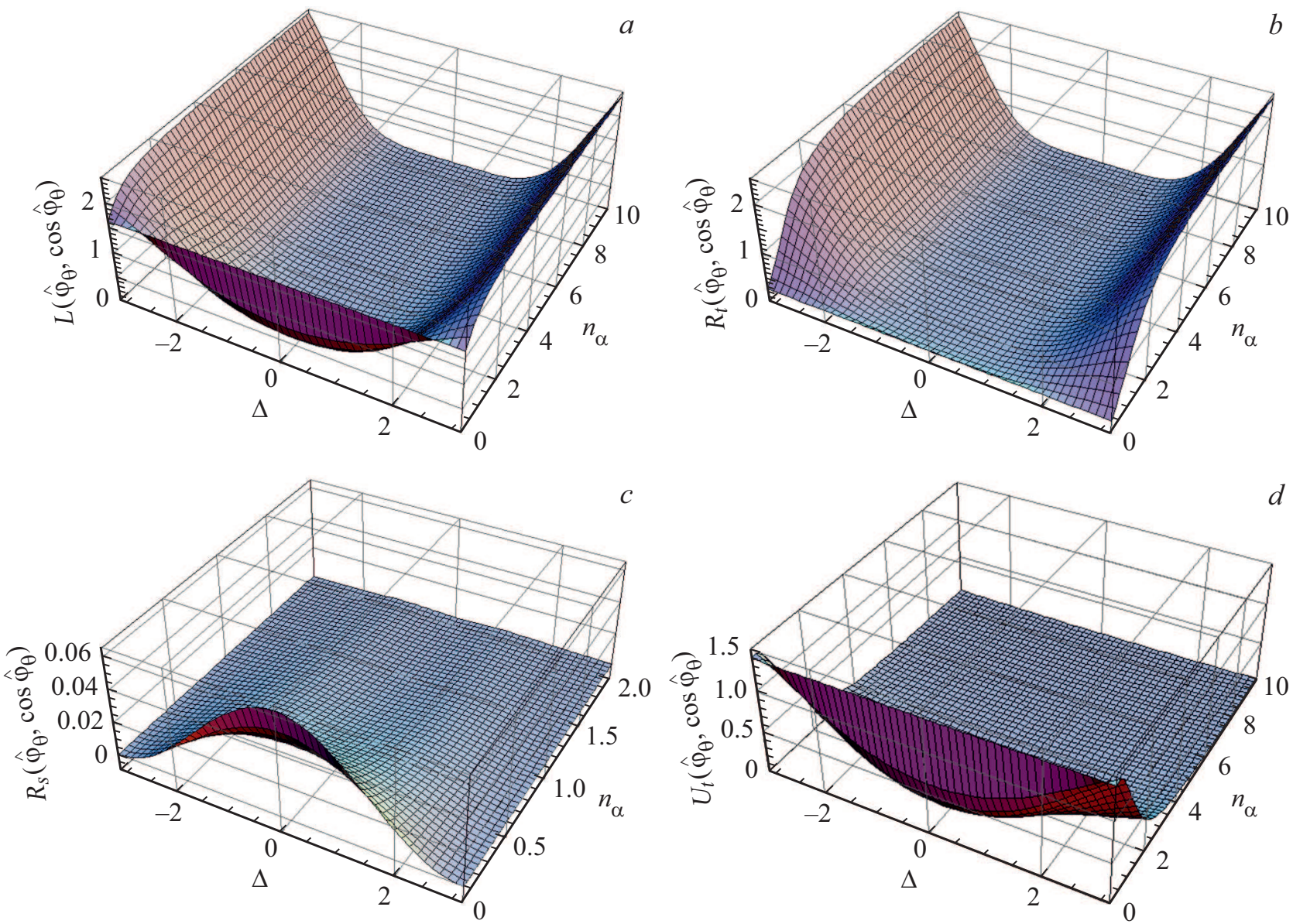

$d$

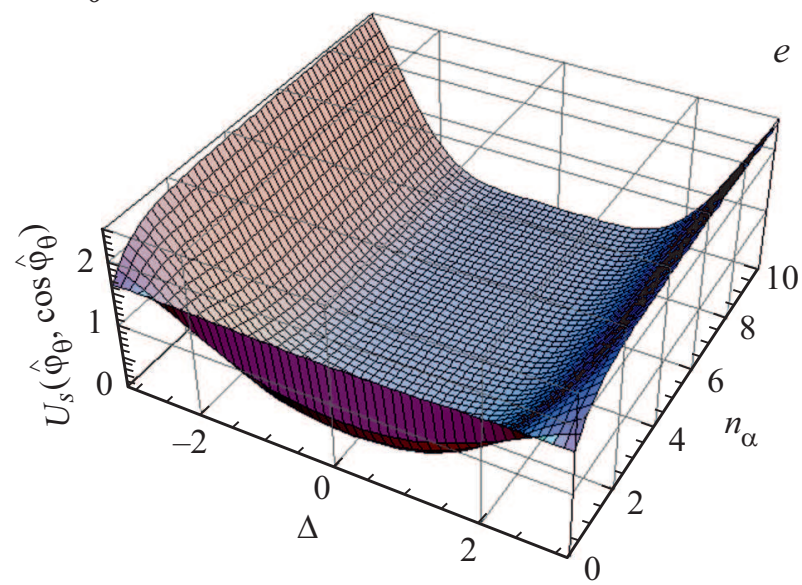

Рис. 3. (a) Зависимость левой части СН $L\left(\hat{\varphi}_{\theta}, \cos \hat{\varphi}_{\theta}\right) \equiv\left\langle\left(\Delta \hat{\varphi}_{\theta}\right)^{2}\right\rangle_{p . s .}\left\langle\left(\Delta \cos \hat{\varphi}_{\theta}\right)^{2}\right\rangle$ от $n_{\alpha} \quad$ и $\Delta \equiv \varphi_{\beta}-\varphi_{\alpha}$ разности фаз когерентных состояний для поля в состоянии фазовой суперпозиции в случае $\xi=0$. (b) Зависимость правой части ЖСН $R_{t}\left(\hat{\varphi}_{\theta}, \cos \hat{\varphi}_{\theta}\right) \equiv\left|\left\langle\hat{\varphi}_{\theta}, \cos \hat{\varphi}_{\theta}\right)^{2}\right\rangle_{p . s .}^{2}$ от $n_{\alpha}$ и разности фаз когерентных состояний $\Delta \equiv \varphi_{\beta}-\varphi_{\alpha}$ для поля в состоянии фазовой суперпозиции в случае $\xi=0, \varphi_{\alpha}=0$. (c) Зависимость правой части ЖСН $R_{s}\left(\hat{\varphi}_{\theta}, \cos \hat{\varphi}_{\theta}\right) \equiv \frac{1}{4}\left|\left\langle\left[\hat{\varphi}_{\theta}, \cos \hat{\varphi}_{\theta}\right]\right\rangle_{p . s .}\right|^{2}$ от $n_{\alpha}$ и разности фаз когерентных состояний $\Delta \equiv \varphi_{\beta}-\varphi_{\alpha}$ для поля в состоянии фазовой суперпозиции в случае $\xi=0, \varphi_{\alpha}=0$. $(d)$ Зависимость разности левой и правой частей ЖСН $U_{t}\left(\hat{\varphi}_{\theta}, \cos \hat{\varphi}_{\theta}\right) \equiv L\left(\hat{\varphi}_{\theta}, \cos \hat{\varphi}_{\theta}\right)-R_{t}\left(\hat{\varphi}_{\theta}, \cos \hat{\varphi}_{\theta}\right)$ от $n_{\alpha}$ и разности фаз когерентных состояний $\Delta \equiv \varphi_{\beta}-\varphi_{\alpha}$ для поля в состоянии фазовой суперпозиции в случае $\xi=0, \varphi_{\alpha}=0$. (e) Зависимость разности левой и правой частей $\mathrm{MCH} U_{s}\left(\hat{\varphi}_{\theta}, \cos \hat{\varphi}_{\theta}\right) \equiv L\left(\hat{\varphi}_{\theta}, \cos \hat{\varphi}_{\theta}\right)-R_{S}\left(\hat{\varphi}_{\theta}, \cos \hat{\varphi}_{\theta}\right)$ от $n_{\alpha}$ и разности фаз когерентных состояний $\Delta \equiv \varphi_{\beta}-\varphi_{\alpha}$ для поля в состоянии фазовой суперпозиции в случае $\xi=0, \varphi_{\alpha}=0$. 


\section{СН для оператора фазы поля и тригонометрических функций оператора фазы поля в состояниях фазовых суперпозиций когерентных состояний}

Для расчетов СН для оператора фазы и косинуса оператора фазы используем следующее выражения для произведения этих операторов:

$$
\begin{aligned}
& \hat{\varphi}_{\theta} \cos \hat{\varphi}_{\theta}=\theta_{0, p} \cos \hat{\varphi}_{\theta}+\frac{\pi}{(S+1)^{2}} \\
& \times\left\{\sum_{m, n, n^{\prime}=0}^{S, S-1, S} m\left[e^{-i\left(n-n^{\prime}\right) \theta_{m}}\left|n^{\prime}\right\rangle\left\langle n+1\left|+e^{-i\left(n-n^{\prime}+1\right) \theta_{m}}\right| n^{\prime}\right\rangle\langle n|\right]\right. \\
& +\sum_{m, n^{\prime}=0}^{S} m\left[e^{\left.i(S+1) \theta_{0, p}+\left(n^{\prime}-S\right) \theta_{m}\right]}\left|n^{\prime}\right\rangle\langle 0|\right. \\
& \left.\left.+e^{\left.-i(S+1) \theta_{0, p}-n^{\prime} \theta_{m}\right]}\left|n^{\prime}\right\rangle\langle S|\right]\right\}
\end{aligned}
$$

где

$$
\begin{gathered}
\theta_{m}=\theta_{0, p}+\frac{2 \pi m}{S+1}, \quad m=0,1, \ldots, S, \\
\theta_{0, p}=\frac{\varphi_{\alpha}+\varphi_{\beta}}{2}-\pi .
\end{gathered}
$$

Среднее квантово-механическое от оператора (65) может быть найдено с помощью формул (53), (54) после суммирования по $m$ и взятия предела $S \rightarrow \infty$ имеет следующий вид:

$$
\begin{aligned}
& \left\langle\hat{\varphi}_{\theta} \cos \hat{\varphi}_{\theta}\right\rangle_{\text {p.s. }}=\theta_{0, p} \cos \hat{\varphi}_{\theta}+\frac{\pi}{(S+1)^{2}}\left(\theta_{0 . p}+\pi\right)\left\langle\cos \hat{\varphi}_{\theta}\right\rangle_{\text {p.s. }} \\
& +\frac{1}{2}\left\{\sum_{n \neq n^{\prime}}^{\infty} \frac{e^{i \theta_{0, p}\left(n-n^{\prime}\right)}}{i\left(n^{\prime}-n\right)} Z_{n^{\prime}}^{*}(\alpha, \beta, \xi) Z_{n+1}(\alpha, \beta, \xi)\right. \\
& \left.+\sum_{n+1 \neq n^{\prime}}^{\infty} \frac{e^{i \theta_{0, p}\left(n^{\prime}-n-1\right)}}{i\left(n^{\prime}-n-1\right)} Z_{n^{\prime}}^{*}(\alpha, \beta, \xi) Z_{n}(\alpha, \beta, \xi)\right\},
\end{aligned}
$$

где использовано $S Z_{S}^{*}(\alpha, \beta, \xi) \rightarrow 0$ при $S \rightarrow \infty, \forall n_{\alpha}$.

Аналогичным путем, вычисляя среднее $\left\langle\cos \hat{\varphi}_{\theta} \hat{\varphi}_{\theta}\right\rangle_{\text {p.s. }}$, находим среднее коммутатора $\left\langle\left[\hat{\varphi}_{\theta}, \cos \hat{\varphi}_{\theta}\right]\right\rangle_{\text {p.s. }}$, определяющее правую часть мягкого $\mathrm{CH}$ (4) при $\hat{A}=\hat{\varphi}_{\theta}$, $\hat{B}=\cos \hat{\varphi}_{\theta}$,

$$
\begin{aligned}
& \left\langle\left[\hat{\varphi}_{\theta}, \cos \hat{\varphi}_{\theta}\right]\right\rangle_{\mathrm{p} . \mathrm{s} .}=i \operatorname{Im} \frac{1}{i} \\
& \times\left\{\sum_{n \neq n^{\prime}}^{\infty} \frac{e^{i \theta_{0, p}\left(n-n^{\prime}\right)}}{n-n^{\prime}} Z_{n+1}^{*}(\alpha, \beta, \xi) Z_{n^{\prime}}(\alpha, \beta, \xi)\right. \\
& \left.+\sum_{n+1 \neq n^{\prime}}^{\infty} \frac{e^{-i \theta_{0, p}\left(n^{\prime}-n-1\right)}}{n-n^{\prime}+1} Z_{n}^{*}(\alpha, \beta, \xi) Z_{n^{\prime}}(\alpha, \beta, \xi)\right\} .
\end{aligned}
$$

Пример зависимости левой части $\mathrm{CH}$ для оператора фазы и косинуса оператора фазы вида $L\left(\hat{\varphi}_{\theta}, \cos \hat{\varphi}_{\theta}\right) \equiv\left\langle\left(\Delta \hat{\varphi}_{\theta}\right)^{2}\right\rangle_{\text {p.s. }}\left\langle\left(\Delta \cos \hat{\varphi}_{\theta}\right)^{2}\right\rangle_{\text {p.s. }}$ от значений $n_{\alpha}$ и разности фаз когерентных состояний $\Delta \equiv \varphi_{\theta}-\varphi_{\alpha}$ для поля в состоянии фазовой суперпозиции вида показан на рис. $3, a$. На рисунке видно, что $L\left(\hat{\varphi}_{\theta}, \cos \hat{\varphi}_{\theta}\right) \approx 0$ для значений $|\Delta|<0.2$ и стремится к величине 2.5 при $n_{\alpha} \gg 1$. Правые части ЖСН и МСН для операторов фазы и косинуса фазы для поля в фазовой суперпозиции когерентных состояний качественно отличаются друг от друга. На рис. $3, b$ и $c$ показаны зависимости правых частей $R_{t}\left(\hat{\varphi}_{\theta}, \cos \hat{\varphi}_{\theta}\right)$ и $R_{s}\left(\hat{\varphi}_{\theta}, \cos \hat{\varphi}_{\theta}\right)$ от значений $n_{\alpha}$ и разности фаз когерентных состояний $\Delta$. Сравнение зависимостей, изображенных на этих рисунках, показывает, что правые части ЖСН и МСН различны при всех $\Delta$, исключая область $|\Delta|$ вблизи 0. Наибольшее отличие имеет место при $|\Delta|$ вблизи $\pi$, где $R_{t}\left(\hat{\varphi}_{\theta}, \cos \hat{\varphi}_{\theta}\right) \gg R_{s}\left(\hat{\varphi}_{\theta}, \cos \hat{\varphi}_{\theta}\right)$ для любых значений $n_{\alpha}$.

Разности левых и правых частей ЖСН и $\mathrm{MCH}$ $U_{x}\left(\hat{\varphi}_{\theta}, \cos \hat{\varphi}_{\theta}\right) \equiv L\left(\hat{\varphi}_{\theta}, \cos \hat{\varphi}_{\theta}\right)-R_{x}\left(\hat{\varphi}_{\theta}, \cos \hat{\varphi}_{\theta}\right), \quad x \equiv t, s$ также качественно различаются для всех $\Delta$ и $n_{\alpha}$, исключая область $|\Delta|$ вблизи 0 . Величина $U_{t}\left(\hat{\varphi}_{\theta}, \cos \hat{\varphi}_{\theta}\right)$ для любых $\Delta$ близка к 0 при $n_{\alpha} \gg 1$, тогда как $U_{s}\left(\hat{\varphi}_{\theta}, \cos \hat{\varphi}_{\theta}\right)$ при $n_{\alpha} \gg 1$ близка к 2.5. Как показано на рис. $3, d, e$, величина стремится к 0 с ростом $n_{\alpha}$ для всех значений параметров, тогда как $U_{x}\left(\hat{\varphi}_{\theta}, \cos \hat{\varphi}_{\theta}\right)$ возрастает до значения 2.5 при $n_{\alpha} \gg 1$ и больших $|\Delta|$.

На основании проведенных расчетов можно сделать вывод о том, что ЖСН для операторов $\hat{\varphi}_{\theta}$ и $\cos \hat{\varphi}_{\theta}$ указывает на то, что состояния фазовых суперпозиций когерентных состояний поля являются квантовыми состояниями с минимальной неопределенностью, тогда как МСH (соотношение Гейзенберга) не позволяет сделать такого вывода.

\section{Заключение}

В настоящей работе нами проведено исследование различных СН для эрмитового оператора фазы $\varphi_{\theta}$ и оператора числа фотонов $\hat{n}$, числа фотонов $\hat{n}$ и оператора $\cos \hat{\varphi}_{\theta}$, а также для оператора фазы $\hat{\varphi}_{\theta}$ и оператора $\cos \hat{\varphi}_{\theta}$ для ЭМ поля в состояниях обобщенных фазовых суперпозиций двух когерентных состояний.

Нами показано, что ЖСН и МСН для числа фотонов и фазы поля $\left(\hat{n}-\hat{n}_{\theta}\right)$ в рассмотренном квантовом состоянии совпадают между собой. При этом состояния фазовых суперпозиций когерентных состояний в общем случае не являются состояниями с минимальной неопределенностью. Условие минимальной неопределенности достигается лишь локально, на узких интервалах изменения разности фаз когерентных состояний $\Delta=\varphi_{\beta}-\varphi_{\alpha}$ для больших средних значений числа фотонов когерентных состояний, $n_{\alpha} \gg 1$.

ЖСН и $\mathrm{MCH}$ для наблюдаемых $\hat{n}-\cos \hat{\varphi}_{\theta}$ значительно различаются между собой при $n_{\alpha} \sim 1$ и почти совпадают для любых $n_{\alpha}$ при $|\Delta| \approx \pi$. Это означает, что в частном случае состояния „шредингеровского кота“ 
поля при $|\Delta|=\pi$ ЖСН и МСН совпадают, и для анализа свойств этих состояний может быть использовано неравенство Гейзенберга. В случае когерентного состояния правые части ЖСН и МСН отличаются значительно при $n_{\alpha} \sim 1$.

Как и в случае $\mathrm{CH} \hat{n}-\hat{\varphi}_{\theta}$, как ЖСН, так и $\mathrm{MCH}$ для $\hat{n}-\cos \hat{\varphi}_{\theta}$ указывают на то, что состояния фазовых суперпозиций не являются в общем случае состояниями с минимальной неопределенностью. Произведение неопределенностей этих величин совпадает с правой частью ЖСН только на ограниченном интервале значений $\Delta$ вблизи $|\Delta|=0$ для $n_{\alpha} \gg 1$, и только в этих условиях рассматриваемые квантовые состояния поля представляют собой состояния с минимальной неопределенностью.

Проведенные нами расчеты показали, что правые части ЖСН и МСН для операторов фазы и косинуса оператора фазы поля $\left(\hat{\varphi}_{\theta}-\cos \hat{\varphi}_{\theta}\right)$ в состояниях фазовых суперпозиций когерентных состояний качественно отличаются между собой. Наибольшее различие имеет место при больших значениях разности фаз когерентных состояний $|\Delta|$, при этом правая часть ЖСН много больше правой части МСН. ЖСН указывает на то, что состояния фазовых суперпозиций когерентных состояний являются состоянием с минимальной неопределенностью (MUS) для $n_{\alpha} \gg 1$, тогда как MCH (неравенство Гейзенберга) показывает значительное различие правых и левых частей при больших значениях $|\Delta| \leq \mathcal{\pi}$ для любых значений $n_{\alpha}$. Это позволяет сделать вывод о том, что при исследовании $\mathrm{CH} \hat{\varphi}_{\theta}-\cos \hat{\varphi}_{\theta}$ состояния „шредингеровского кота“" $(|\Delta|=\pi)$ необходимо рассмотрение ЖСН.

Использование ЖСН для операторов $\hat{n}-\cos \hat{\varphi}_{\theta}$ также необходимо для анализа рассматриваемых нами состояний поля при малых числах фотонов $\left(n_{\alpha} \sim 1\right)$ в случае, когда разности фаз когерентных состояний $|\Delta|$ заметно отличаются от $\pi$.

\section{Конфликт интересов}

Авторы заявляют, что у них нет конфликта интересов.

\section{Список литературы}

[1] Holland M.J., Burnett K. // Phys. Rev. Lett. 1993. V. 71. N 9. P. $1355-1358$.

[2] Schnabel Roman. // Phys. Rep. 2017. V. 684. P. 1-51.

[3] Demkowicz-Dobrzański R., Jarzyna M., Kołodyński J. // Progress in Optics. 2015. V. 60. P. 345-435.

[4] Barnett S.M., Pegg D.T. // J. Mod. Opt. 1989. V. 36. N 9. P. 7-19.

[5] Pegg D.T., Barnett S.M. // Phys. Rev. A. 1989. V. 39. N 4. P. 1665-1675.

[6] Lynch R. // Phys. Rev. A. 1990. V. 41. N 5. P. 2841-2843.

[7] Popov V.N., Yarunin V.S. // J. Mod. Optics. 1992. V. 39. N 7. P. $1525-1531$

[8] Lynch R. // Phys. Rep. 1995. V. 256. N 9. P. 367-436.

[9] Vogel W., Welsch D.-G. Quantum Optics. 3-d edn. WileyVCH, 2006. 508 p.
[10] Gronbech-Jensen N., Christiansen P.L., Ramanujam P.S. // J. Opt. Soc. Am. B. 1989. V. 6 (12). P. 2423-2429.

[11] Mendas I., Popovic D.B. // Phys. Rev. A. 1995. V. 50. N 9. P. 947-953.

[12] Opatrny T. // J. Phys. A: Math. Gen. 1995. V. 28. N 7. P. 6961-6975.

[13] Vaccaro J.A., Pegg D.T. // J. Mod. Optics. 1990. V. 37. N 1. P. 17-39.

[14] Yurke B., Stoler D. // Phys. Rev. Lett. 1986. V. 57. N 1. P. 13-16.

[15] Yurke B., Stoler D. // Phys. Rev. A. 1987. V. 35. N 11. P. 4846-4849.

[16] Schleich W., Pernigo M., Fam Le Kein. // Phys. Rev. A. 1991. V. 44. N 3. P. 2172-2187.

[17] Buzek V., Wilson-Gordon A.D., Knight P.L., Lai W.K. // Phys. Rev. A. 1992. V. 45. N 11. P. 8079-8094.

[18] Gurraway B.M., Knight P.L. // Phys. Scr. 1993. V. 48. P. 66-76; Phys. Rev. A. 1992. V. 46 (9). P. R5346-5349.

[19] Buzek V., Gantsog T.S., Kim M.S. // Phys. Scr. 1993. V. 48. P. $131-142$

[20] Smithey D.T., Beck M., Raymer M.G., Faridani A. // Phys. Rev. Lett. 1993. V. 70 (9). P. 1244-1247.

[21] Smithey D.T., Beck M., Cooper J., Raymer M.G., Faridani A. // Phys. Scr. 1993. V. 48. P. 35-44.

[22] Beck M, Smithey D.T., Cooper J., Raymer M.G. // Opt. Lett. 1993. V. 18 (15). P. 1259-1261.

[23] Козловский А.В. // Опт. и спектр. 2016. Т. 120. N 44. C. 626-635; Kozlovskii A.V. // Opt. Spectrosc. 2016. V. 120 (4). P. 626-635.

[24] Kozlovskii A.V. // J. Mod. Optics. 2016. V. $63(21)$. P. 2356-2366.

[25] Kozlovskii A.V. // J. Mod. Optics. 2019. V. 66 (5). P. 463-472. 